\title{
Two rhodamine lactam modulated lysosome-targetable fluorescence probes for sensitively and selectively monitoring subcellular organelle $\mathrm{pH}$ change
}

Hongmei Li, ${ }^{\mathrm{I},}{ }^{\dagger}$ Cuiling Wang,,${ }^{\mathrm{II}}{ }^{\dagger}$ Mengyao She, ${ }^{\mathrm{I}}$ Yuelu Zhu, ${ }^{\mathrm{I}}$ Jidong Zhang, ${ }^{\mathrm{I}}$ Zheng Yang, ${ }^{\mathrm{I}}$ Ping Liu, ${ }^{, I}$ Y Yaoyu Wang, ${ }^{\text {I }}$ Jianli $\mathrm{Li}^{*}$, I

${ }^{\text {I }}$ Ministry of Education Key Laboratory of Synthetic and Natural Functional Molecule Chemistry, College of Chemistry \& Materials Science, Northwest University, Xi'an 710069, China

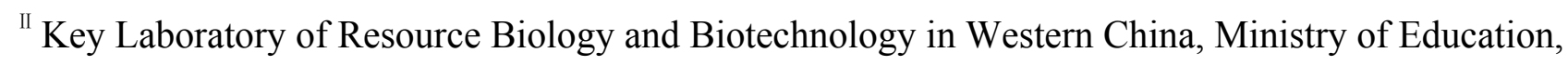
College of Life Science, Northwest University, Xi'an 710069, China

*Jianli Li. E-mail: lijianli@nwu.edu.cn, *Ping Liu. E-mail: liuping@nwu.edu.cn. Tel:+86-02981535026; fax: +86-29-88308396.

${ }^{\dagger}$ These authors contributed equally to this paper.

ABSTRACT: Be a powerful technique for convenient detection of $\mathrm{pH}$ change in living cells, especially at subcellular level, fluorescent probes has attracted more and more attention. In this work, we designed and synthesized three rhodamine lactam modulated fluorescent probes RS1, RS2 and RS3, which all respond sensitively toward weak acidity (pH range 4-6) via the photophysical property in buffer solution without interference from the other metal ions, and they also show ideal pKa values and excellent reversibility. Particularly, by changing the lone pair electrons distribution of lactam- $\mathrm{N}$ atom with different conjugations, RS2 and RS3 exhibit high quantum yield, negligible cytotoxicity and excellent permeability. They are suitable to stain selectively lysosomes of tumor cells and monitor its $\mathrm{pH}$ changes sensitively via optical molecular imaging. The above findings suggest that the probes we designed could 
act as ideal and easy method for investigating the pivotal role of $\mathrm{H}^{+}$in lysosomes and are potential $\mathrm{pH}$ detectors in disease diagnosis through direct intracellular imaging.

Keywords: Rhodamine lactam; Fluorescence probe; conjugation effect; Lysosome-targetable; Bioimaging.

\section{Introduction}

Lysosomes are advanced organelles found in virtually all eukaryotic cells and contain more than 60 acidic hydrolases, cathepsins, and many specific membrane proteins [1-4]. Lysosomes not only promote the degradation of macromolecules in cellular metabolism, but also are involved in more specialized secretory functions $[5,6]$. The acidic microenvironment in the cytoplasm is provided by lysosomes, which are responsible for the more acidic lysosomal lumen of cancer cells ( $\mathrm{pH} 4.5-5.5 \mathrm{vs}$ 5.0-6.0 in normal cells) [7]. The investigation of the spatial-temporal distribution of $\mathrm{pH}$ and its fluctuations in lysosomes, especially those in cancer cells, provides a strategic approach to visualize the early development of tumor tissue [8]. It is obvious that exploring simple and convenient ways to study the lysosomal lumen of cancer cells is desirable for cancer research [9-12].

Fluorescent probes have attracted continuing attention due to the simplicity and high sensitivity of fluorescence detection. Most of the traditional lysosomal targeted probes, consisting of a luminescent core and a neighboring amine side chain, rely on protonation mediated inhibition of the intramolecular PET (photo-induced electron transfer) process to induce fluorescence quenching (Figure 1) [13-16]. However, the fluorescence quenching of those probes was always inadequate which tends to cause high background fluorescence signals inside cells [17-18]. In addition, some of those probes also have disadvantages of short absorption and emission wavelengths which can easily lead to cell damage. However, being a fluorescence-enhanced probe, rhodamine-lactams operate in an "off-on" fashion, where at neutral and alkaline conditions the compounds are in "off" style and the fluorescent is silent, while easily isomerize 
into the "on" style via protonation opening of the intramolecular lactam ring with fully fluorescent and obvious color variation at acid condition [19-21]. Moreover, the rhodamine framework has particular photophysical advantages in biological applications because of its high fluorescence quantum yield, relatively long absorption and emission wavelengths elongated to the visible region, large absorption coefficient and great photostability [22-25]. Furthermore, it can easily avoid "alkalizing effect" of the aliphatic amino which would increase the lysosomal pH after long incubation with the probe [26]. Therefore, rhodamine framework is popular for many researchers in studying the pH of lysosomes [2731]. Considering those facts, three novel rhodamine B-based $\mathrm{pH}$ fluorescent probes RS1, RS2 and RS3 were designed and synthesized. (Scheme.1)

Herein, we introduce a functional group of 2,6-diaminopyridine and then protected the amine side chain with a flexible Schiff base or a rigid naphthyridine to change its properties. A compound containing amine will not only has good solubility in both water and lipid, but also helps the probe to accumulate selectively in an acidic environment, such as a lysosome [32-34]. In addition, the pyridine ring tends to have excellent biocompatibility. The analytical performances of the designed probes were investigated concerning sensitivity, selectivity, reversibility, cell permeability, and cytotoxicity. By introducing different conjugated systems into the recognition groups, the lone pair electrons distribution of lactam$\mathrm{N}$ atom in RS2 and RS3 was changed, which lead to the two probes displayed distinguished optical properties both in vitro and in vivo and suitable for imaging acidic organelles such as tumor lysosomes. (Table S1)

\section{Experimental}

\subsection{General methods and reagents.}


NMR spectra were recorded on the Varian Inova-400MHz spectrometer (at $400 \mathrm{MHz}$ for ${ }^{1} \mathrm{H}$ and 100 $\mathrm{MHz}$ for ${ }^{13} \mathrm{C}$ ) by using tetramethylsilane (TMS) as internal standard. Mass spectra were performed on Model AXIMA-CFR_plus MALDI-TOF Mass Spectroscopy. Absorbance spectra measurements were collected on a Shimadzu UV-1700 spectrophotometer. Fluorescence measurements were performed on a HITACHI F-4500 fluorescence spectrophotometer with a slit width of $5 \mathrm{~nm}$ for excitation and $5 \mathrm{~nm}$ for emission, respectively. X-ray crystal data were collected on Bruker Smart APEX II CCD diffractometer. All pH measurements were made with a Model EL20 meter (METTLER-TOLEDO INSTRUMENTS (SHANGHAI) CO, LTD). The cytotoxicity were analyzed on a Molecular Devices spectra Max-190 microplate reader. The fluorescence images of cells for the staining experiments were performed with an Olympus FV1000 confocal microscope.

Unless otherwise stated, all reagents and solvents used were of analytical grade without further purification. Analytical thin layer chromatography was performed using Merck 60 GF254 silica gel (precoated sheets, $0.25 \mathrm{~mm}$ thick). Silica gel $(0.200-0.500 \mathrm{~mm}, 60 \mathrm{~A}$, J\&K Scientific Ltd.) was used for column chromatography. Double distilled water was used throughout the experiment.

\subsection{Synthesis.}

The detailed synthetic procedures and compound characterizations are mentioned in the Supporting Information.

\subsection{General procedure.}

Sensor RS1, RS2 and RS3 stock solution was prepared in $500 \mu \mathrm{M}$ concentration, respectively. The solutions of the ions were also performed with the salts including $\mathrm{LiCl}, \mathrm{NaCl}, \mathrm{KCl}, \mathrm{CaCl}_{2}, \mathrm{MgCl}_{2}, \mathrm{PbCl}_{2}$, $\mathrm{BaCl}_{2}, \mathrm{CoCl}_{2}, \mathrm{CdCl}_{2}, \mathrm{HgCl}_{2}, \mathrm{CuCl}_{2}, \mathrm{AgNO}_{3}, \mathrm{CrCl}_{3}, \mathrm{MnCl}_{2}, \mathrm{AlCl}_{3}, \mathrm{ZnCl}_{2}, \mathrm{FeCl}_{3}, \mathrm{NiCl}_{2}$, and $\mathrm{FeCl}_{2}$ in 
buffer solution. The Absorptions were recorded at $560 \mathrm{~nm}$ and the fluorescence intensity were recorded at $590 \mathrm{~nm}$ for the three probes with the excitation wavelength at $520 \mathrm{~nm}$.

\subsection{Crystal growth and conditions.}

White single crystals of RS1 and yellow single crystal of RS3 were obtained at room temperature from the mixed solvents of n-hexane-dichloromethane solution by slow evaporation, and the white single crystal of RS2 was obtained from the mixed solvents of acetonitrile-dichloromethane solution. (Figure S1, Figure S2, and Figure S3) Then they were mounted on the goniometer of single crystal diffractometer. The crystal data were collected at $296 \mathrm{~K}$ by using Mo $K \alpha$ radiation by using $\varphi / \omega$ scan mode and collected for Lorentz and polarization effect (SADABS). The structures were solved using the direct method and refined by full-matrix leastsquares fitting on F2 by SHELX-97.

\subsection{Cell culture.}

A549 cells, human lung carcinoma, were grown in Dulbecco's modified Eagle's medium (DMEM) supplemented with 10\% heat-inactivated Fetal bovine serum (FBS) and penicillin(100 units $\left.\mathrm{mL}^{-1}\right)$, streptomycin $\left(100 \mathrm{mg} \mathrm{mL}^{-1}\right)$ at $37{ }^{\circ} \mathrm{C}$ in humidified environment containing $5 \% \mathrm{CO}_{2}$. Cells were seeded on a $\$ 35 \mathrm{~mm}$ confocal dish or 96 -well plate at a density of $2 \times 10^{4}$ Cells mL-1 in medium incubating for $24 \mathrm{~h}$.

\subsection{Confocal Laser Scanning Microscope (CLSM) imaging.}

Before each treatment, A549 cells were washed 3 times with PBS buffer (10 mM, pH 7.4) to remove the residue, and subsequently A549 cells were treated with different concentrations of probes for $0.5-4$ $\mathrm{h}$ at $37^{\circ} \mathrm{C}$ and then the fluorescence of the probes within the cells was analyzed by confocal fluorescence microscopy. LysoTracker Green DND-26 (the green emission) in 504/511 nm (Ex/Em) was collected 
using an excitation wavelength of $488 \mathrm{~nm}, \mathrm{RS} 1, \mathrm{RS} 2$ and RS3 (the red emission) in 560/593 nm (Ex/Em) were collected using an excitation wavelength of $543 \mathrm{~nm}$.

\subsection{Cytotoxicity assay.}

The viability of A549 cells under different treatments were evaluated by performing 3-(4,5dimethylthiazol-2-yl)-2,5-diphenyl-tetrazolium bromide (MTT) assay. Cells $\left(1 \times 10^{5} /\right.$ well) were seeded into a 96-well plate with $100 \mu \mathrm{L}$ medium and culturing for $24 \mathrm{~h}$ at $37^{\circ} \mathrm{C}$ in humidified environment containing 5\% CO2, followed by washing with $100 \mu \mathrm{L} /$ well phosphate buffered saline (PBS) and then cells treating with different concentrations $(0 \mu \mathrm{M}, 0.312 \mu \mathrm{M}, 0.625 \mu \mathrm{M}, 1.25 \mu \mathrm{M}, 2.5 \mu \mathrm{M}, 5 \mu \mathrm{M}, 10 \mu \mathrm{M}$, $20 \mu \mathrm{M}, 40 \mu \mathrm{M}$ ) of probes RS1, RS2 and RS3 for another $6 \mathrm{~h}$. To test the toxicity of LysoTracker Green DND-26, cells were also treated with different concentrations $(0 \mu \mathrm{M}, 0.312 \mu \mathrm{M}, 0.625 \mu \mathrm{M}, 1.25 \mu \mathrm{M}, 2.5 \mu \mathrm{M}$, $5 \mu \mathrm{M}, 10 \mu \mathrm{M}, 20 \mu \mathrm{M}, 40 \mu \mathrm{M})$ of LysoTracker Green DND-26 for $6 \mathrm{~h}$. Cells in culture medium without any

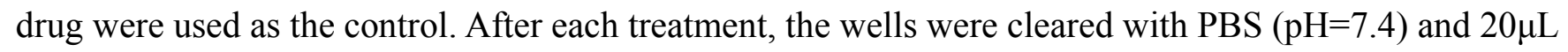
MTT $\left(0.5 \mathrm{mg} \mathrm{ml}^{-1}\right)$ and $180 \mu \mathrm{L}$ medium was added to each well and incubated for an additional $4 \mathrm{~h}$. The medium was then carefully removed, and the purple products were lysed in $200 \mu \mathrm{L}$ dimethyl sulfoxide (DMSO). The plate was shaken for $10 \mathrm{~min}$ at $37^{\circ} \mathrm{C}$ and the absorbance was measured at $490 \mathrm{~nm}$ using a microplate reader. Cell viability was expressed as a percentage of the control culture value. All treatments are repeated 3 times.

\section{Results and discussion}

Sensors RS1, RS2 and RS3 were easily obtained as non-fluorescent products. As shown in Scheme 1, those probes were designed based on the transformation in structure between spirocyclic (nonfluorescent) and ring-opening (strong-fluorescent) forms responding to $\mathrm{H}^{+}$. All the structures of probes and intermediates were confirmed by ${ }^{1} \mathrm{H}$ NMR, ${ }^{13} \mathrm{C}$ NMR, and ESI-MS. The Single crystal of the three probes were obtained and showed in Figure S24. Which clearly revealed the spirolactam unit in the solid 
state, and what is interestingly is that the xanthene ring is nearly perpendicular with the lactam ring. $\left({ }^{13} \mathrm{C}\right.$ NMR spectrum of the three compounds also showed the characteristic signals of the spirolactam moiety at $\delta=66.85 \mathrm{ppm}$. (Figure S16b, Figure S18b, Figure S19b))

With the targeted probes, an optimized Britton-Robinson buffer solution was selected for the spectroscopic investigation based on the acid-base dependency assay at room temperature. Under basicneutral condition, the solution of RS1, RS2 and RS3 were almost colorless and non-fluorescent, however, as shown in Figure S4 and Figure S5, with decreasing the $\mathrm{pH}$ from 7 to 3.67, those probes exhibit a sharp absorption peak at $560 \mathrm{~nm}$, and remarkable fluorescence emission peak at $590 \mathrm{~nm}$ with the solution changed from colorless to pink. The fluorescence signals of RS1 increased with about 62-flod, of RS2 and RS3 with about 76-flod. The effect is mainly due to the addition of $\mathrm{H}^{+}$lead to the $\mathrm{N}$ atom of the lactam being protonated, which generated a delocalization of electrons in xanthene (as shown in scheme 1). The fluorescence titration curves are directly fitted by the relative ratio of fluorescence emission intensities $\left(\mathrm{I} / \mathrm{I}_{\max }\right)$ at their maximum emission peak versus $\mathrm{pH}$ (Figure 2). Interestingly, a good linearity relationship between the ratio $\left(\mathrm{I} / \mathrm{I}_{\max }\right)$ and $\mathrm{pH}$ can be easily obtained. The large straight line slope for RS1, RS2 and RS3 could be found in the $\mathrm{pH}$ range from 4.34 to $5.35,4.62$ to 5.13 and from 4.16 to 5.25 , respectively. Which indicates that those probes are highly sensitive to $\mathrm{pH}$ under weak acid conditions (even for quantitative lysosome pH measurements). Moreover, as shown in Figure S6, the newly designed probes display ideal pKa values which match well with the lysosome $\mathrm{pH}$ of tumor cells (respectively 5.00, 5.00, 4.74 via the Henderson-Hasselbalch-type mass action equation) [35]. Concomitantly, by increasing the size of the conjugate system, the fluorescent quantum yields of RS2 and RS3 were much higher than RS1 at $\mathrm{pH} 4.5 .(\Phi \mathrm{RS} 1=0.37, \Phi \mathrm{RS} 2=0.64, \Phi \mathrm{RS} 3=0.68$, rhodamine $\mathrm{B}$ as a standard $)$ [36]. All above indicated that those probes have promising properties to study the lumen of lysosomes in biological systems. 
To the best of our knowledge, nitrogen and oxygen of spirocyclic can bind to many metal ions in solution, which can also cause the intramolecular lactam ring to open [37-40]. Considering the practical application of the tree sensors, the fluorescence intensity in the presence of $\mathrm{H}^{+}$was compared to other metal ions including $\mathrm{K}^{+}, \mathrm{Na}^{+}, \mathrm{Ca}^{2+}, \mathrm{Mg}^{2+}, \mathrm{Pb}^{2+}, \mathrm{Ba}^{2+}, \mathrm{Co}^{2+}, \mathrm{Cd}^{2+}, \mathrm{Hg}^{2+}, \mathrm{Cu}^{2+}, \mathrm{Ag}^{+}, \mathrm{Cr}^{3+}, \mathrm{Mn}^{2+}, \mathrm{Al}^{3+}, \mathrm{Zn}^{2+}$, $\mathrm{Fe}^{3+}, \mathrm{Ni}^{2+}$ and $\mathrm{Fe}^{2+}$ at different $\mathrm{pH}$ to study the selectivity of RS1, RS2, RS3. As shown in Figure 3, there was no obvious change of fluorescence upon the addition of various metal ions, despite high concentrations of metal ions as a function of its distribution in vivo combined with $\mathrm{pH}$ 7.00. Similarly, at $\mathrm{pH} 4.20$, it is seems that various metal ions did not interfere with the fluorescence changes caused by $\mathrm{H}^{+}$. The results of selectivity experiments of those sensors are almost the same. Therefore, all of the probes can detect $\mathrm{pH}$ changes with high selectivity to the maximum emission fluorescence intensity at $\mathrm{H}^{+}$shown as dramatic fluorescence changes.

As shown in Figure S7, the sensitivity of RS1, RS2 and RS3 to $\mathrm{H}^{+}$was also tested by measuring the fluorescence response within $20 \mathrm{~min}$. (images were recorded at intervals of $2 \mathrm{~s}$ ) Once they were placed in the buffer solution, the fluorescence intensity of RS2 can reach the maximum within 1 minutes, and RS1, RS3 in about $200 \mathrm{~s}$, then they all maintained a stable value both at $\mathrm{pH} 4.26$ and $\mathrm{pH} 5.35$. But no significant changes in the fluorescence intensity were recorded at pH 7.0. Compared RS2 with RS1 and RS3, it seems that RS2 has the shortest response time, which perhaps owes it to the rigid structure of naphthyridine. According to the above recorded data, the developed sensors are not only sensitive to $\mathrm{H}^{+}$, but also can provide a reliable stable signal. To explore the reversibility, the $\mathrm{pH}$ of the buffer solution was modulated several times between weakly acid and neutral conditions (Figure S8). The maximum fluorescence intensity of RS1, RS2 and RS3 under these conditions recovered almost the original values. Which indicates that they can detect conveniently weakly acidic conditions with an ideal reversibility. Meanwhile, a possible mechanism of the reversibility for probe RS2 under weak acidic condition was proposed in 
Figure 4 and ESI-MS, ${ }^{1} \mathrm{H}$ NMR spectroscopy were also used for purpose of proving the mechanism. (Figure S9 and S10)

In order to investigate the further application of RS1, RS2 and RS3 in vivo, an experiment about cell permeability and $\mathrm{pH}$ sensitively is necessary. Confocal microscopy images of A549 cells were used to characterize the $\mathrm{pH}$ imaging. A549 cells were firstly incubated with different concentrations of sensors for $0.5-4 \mathrm{~h}$. As shown in Figure S12, after $0.5 \mathrm{~h}$ of incubation with RS2 at $37{ }^{\circ} \mathrm{C}$ in humidified environment containing 5\% $\mathrm{CO}_{2}$ air incubator, very clear images of the A549 cells were obtained with a very low concentration $(4 \mu \mathrm{M})$, in the same condition, we can easily observe from Figure S11 and Figure S13 that RS1 and RS3 would have bigger concentration about $10 \mu \mathrm{M}$ and $6 \mu \mathrm{M}$ respectively in $0.5 \mathrm{~h}$. This indicates that RS2 is a more promising fluorescence probe than RS1 and RS3 with good cell permeability and desirable $\mathrm{pH}$ sensitivity in vivo. For further confirmation of the subcellular distribution of the three probes, a commercially available lysosome specific staining probe LysoTracker Green DND-26 was used to co-stain A549 cells. The clear green signals could be collected from the LysoTracker Green and red signals from the probes RS1, RS2 and RS3. The excellent function of co-localization with lysosome was confirmed by the merged images showed convincing yellow fluorescence. (Figure S14 for RS1, Figure 5 for RS2 and Figure S15 for RS3) The above experimental results suggest that all of the probes can selectively stain lysosomes in living cells with different concentrations and response times.

To further study the sensitivity and real-time monitoring capacity of the probes in vivo, another commercial reagent was used. Bafilomycin A1 (Baf A1), a selective inhibitor of the vacuolar-type $\mathrm{H}^{+}-$ ATPase (V-ATPase), which can disrupt the acidic environment of the lysosomes and increase the inner $\mathrm{pH}[41,42]$. A549 cells were pretreated with different concentrations of bafilomycin A1 for $5 \mathrm{~h}$, then 10 $\mu \mathrm{M}$ RS1, $10 \mu \mathrm{M}$ RS2, $10 \mu \mathrm{M}$ RS3, and 0.5 $\mu \mathrm{M}$ LysoTracker Green DND-26 were used to stain the used cells respectively for another $2 \mathrm{~h}$. The results showed in Figure 6 indicate that compared to commercial 
dye, RS2 and RS3 can sensitively respond to lysosomal $\mathrm{pH}$ changes with the fluorescence intensity gradually weakened. Probe RS1 performed inferior to RS2, RS3 and LysoTracker. The results demonstrate that probes RS2 and RS3 can be used for real-time monitoring of lysosomal pH changes sensitively during physiological and pathological processes.

Furthermore, the cytotoxicity of RS1, RS2, RS3 and commercial dye (Lyso Tracker green DND-26) on the A549 cell line were studied by MTT assays (Figure 7). After $6 \mathrm{~h}$ of cellular co-cultured, no obvious toxicity was found for RS2, RS3 and commercial dyes in cells at our experimental conditions, Unexpectedly, the high level (above $10 \mu \mathrm{M}$ ) of RS1 was highly toxic to the cells. Despite the latter, the results suggest that our probes are suitable for biological applications.

\section{Conclusions}

In summary, we have designed and synthesized three novel $\mathrm{pH}$ fluorescent probes with a long excitation wavelength by introducing the rhodamine fluorophore. All new probes exhibit high sensitivity, sufficient selectivity and excellent reversibility. In addition, the probes could be used as lysosome targeted probes in A549 cells with ideal pKa values. In particular, by modifying the amino side groups of RS1 with a flexible or a rigid conjugated system, the lone pair electrons distribution of the $\mathrm{N}$ atom in the lactam was changed obviously, which benefit the probes RS2 and RS3 performed best with high quantum yield, very low cytotoxicity, excellent cell membrane permeability and good sensitivity for staining lysosomes in vivo. The series of the results show that our probes would be benefit to understand the acid environment of lysosomes in related cells and organs with potential biological significance. Furthermore, these probes may be promising new tools to visualize tumor cells.

\section{Acknowledgements}


We are grateful for the support from the NSF of China (No. 21272184, 21103137, J1210057), the Shaanxi Provincial Natural Science Fund Project (No. 2015JZ003), the Xi'an City Science and Technology Project (No. CXY1429(6), CXY1434(7)), the Northwest University Science Foundation for Postgraduate Students (No. YZZ13042, YZZ14052).

\section{Appendix A. Supplementary data}

Supplementary data associated with this article can be found, in the online version.

\section{References}

[1] H. Appelqvist, p. Waster, K. Kagedal, K. Ollonger, The lysosome: from waste bag to potential therapeutic target. J. Mol. Cell. Boil. 5 (2013) 214-226.

[2] J.P. Luzio, P.R. Pryor, N.A. Bright, Lysosomes: fusion and function. Nat. Rev. Mol. Cell. Boil. 8 (2007) 622-632.

[3] D.F. Bainton, The discovery of lysosomes. J. Cell Biol. 91 (1981) 66s-76s.

[4] P. Saftig, J. Klumperman. Lysosome biogenesis and lysosomal membrane proteins: trafficking meets function. Nat. Rev. Mol. Cell. Biol. 10 (2009) 623-635.

[5] C. Watts, The endosome-lysosome pathway and information generation in the immune system. Biochim. Biophys. Acta. 1824 (2012) 14-21.

[6] H. Zhao, Membrane trafficking in osteoblasts and osteoclasts: new avenues for understanding and treating skeletal diseases .Traffic. 13 (2012) 1307-1314.

[7] S. Ohkuma, B. Poole, Fluorescence probe measurement of the intralysosomal pH in living cells and the perturbation of pH by various agents. Proc. Natl. Acad. Sci .75 (1978) 3327-3331.

[8] H. Lee, W. Akers, K.Bhushan, S. Bloch, G. Sudlow, R. Tang, S. Achilefu, Near-infrared pHactivatable fluorescent probes for imaging primary and metastatic breast tumors. Bioconjug. Chem. 22 (2011) 777-784. 
[9] X.X. Zhao, X.P. Chen, S.L. Shen, D.P. Li, S.Zhou, Z.Q. Zhou, Y.H. Xiao, G. Xi, J.Y. Miao, B.X. Zhao, A novel pH probe based on a rhodamine-rhodamine platform. RSC Adv. 4 (2014) 50318-50324.

[10] K.K. Yu, K. Li, J.T. Hou, H.H. Qin, Y.M. Xie, C.H. Qian, X.Q. Yu, Rhodamine-based lysosometargeted fluorescence probes: high $\mathrm{pH}$ sensitivity and their imaging application in living cells. RSC Adv. 4 (2014) 33975-33980.

[11] M.H. Lee, J.H. Han, J.H. Lee, N. Park, R. Kumar, C. Kang, J.S. Kim, Two-Color Probe to Monitor a Wide Range of pH Values in Cells. Angew. Chem. Int. Ed. 52 (2013) 6206-6209.

[12] J.L. Hu, F. Wu, S. Feng, J.H. Xu, Z.H. Xu, Y.Q. Chen, T. Tang, X.C. Weng, X. Zhou, A convenient ratiomeric $\mathrm{pH}$ probe and its application for monitoring $\mathrm{pH}$ change in living cells. Sens Actuators $\mathrm{B}$ chem. 196 (2014) 194-202.

[13] H.J. Lin, P. Herman, J.S. Kang, J.R. Lakowicz, Fluorescence lifetime characterization of novel low-pH probes. Anal. Biochem. 294 (2001) 118-125.

[14] C. Sandrine, M. Adrien, A. Damien, C.L. Emmanuelle, A. Jean-Francois, J. Ludovic, L. Annie, Reactant Concentrations from Fluorescence Correlation Spectroscopy with Tailored Fluorescent Probes. An Example of Local Calibration-Free pH Measurement. J. Am. Chem. Soc. 127 (2005) 15491-15505.

[15] C. Sandrine, R. Odile, B. Jean-Bernard, A. Damien, A. Jean-Francois, M. Adrien, L. Jullien, pH sensors: An efficient fluorescent probe for ratiometric $\mathrm{pH}$ measurements in aqueous solutions. Angew. Chem. Int. Ed. 43 (2004) 4785-4788.

[16] U. Yasuteru, A. Daisuke, H. Yukihiro, K. Yoshinori, B. Tristan, K. Mako, N. Tetsuo, W. Toshiaki, H. Akira, L.C. Peter, K. Hisataka, Selective molecular imaging of viable cancer cells with pH-activatable fluorescence probes. Nat. Med. 15 (2009) 104-109.

[17] K. Rurack, Flipping the light switch 'ON' - the design of sensor molecules that show cation-induced fluorescence enhancement with heavy and transition metal ions. Spectrochim. Acta. A. Mol. Biomol. Spectrosc. 57 (2001) 2161-2195. 
[18] K. Rurack, U. Resch-Genger, Rigidization, preorientation and electronic decoupling-the 'magic triangle' for the design of highly efficient fluorescent sensors and switches. Chem. Soc. Rev. 31 (2002) 116-127.

[19] S.L. Shen, X.P. Chen, X.F. Zhang, J.Y. miao, B.X. Zhao, A rhodamine B-based lysosomal pH probe, J. Mater. Chem. B. 3 (2015) 919-925.

[20] X.F. Zhang, T. Zhang, S.L. Shen, J.Y. Miao, B.X. Zhao, A ratiometric lysosomal pH probe based on the coumarin-rhodamine FRET system, RSC Adv. 5 (2015) 49115-49121.

[21] Z. Li, Z.W. Xue, Z.S. Wu, J.H. Han, S.F. Han, Chromo-fluorogenic detection of aldehydes with a rhodamine based sensor featuring an intramolecular deoxylactam. Org. Biomol. Chem. 9 (2011) 76527654

[22] Z.W. Ye, Y. Xiao, H.Y. Guo, Chao Wang, Specific and photostable rhodamine-based tracker for 3D video imaging of single acidic organelles. RSC Adv. 4 (2014), 35547-37555.

[23] H.S. Lv, J. Liu, J. Zhao, B.X. Zhao, J.Y. Miao, Highly selective and sensitive pH-responsive fluorescent probe in living Hela and HUVEC cells. Sens. Actuators B Chem. 177 (2013) 956-963.

[24] Q.A. Best, R. Xu, M.E. McCarroll, L. Wang, D.J. Dyer, Design and Investigation of a Series of Rhodamine-Based Fluorescent Probes for Optical Measurements of pH. Org. Lett. 12 (2010) 3219-3221. [25] X.L. Chen, X.M. Meng, S.X. Wang, Y.L. Cai, Y.F. Wu, Y. Feng, M.Z. Zhu, Q.X. Guo, A Rhodamine-based fluorescent probe for detecting $\mathrm{Hg}^{2+}$ in a fully aqueous environment. Dalton Trans. 42 (2013) 14819-14825.

[26] H.S. Lv, S.Y. Huang, B.X. Zhao, J.Y. Miao, A new rhodamine B-based lysosomal pH fluorescent indicator. Anal. Chim. Acta. 788 (2013) 177-182.

[27] H.S. Lv, S.Y. Huang, Y. Xu, X. Dai, J.Y. Miao, B.X. Zhao, A new fluorescent pH probe for imaging lysosomes in living cells. Bioorg. Med. Chem. Lett. 24 (2014) 535-538. 
[28] R. Huang, S.Y. Yan, X.L. Zheng, F.L. Luo, M.G. Deng, B.S. Fu, Y.J. Xiao, X. Zhao, X. Zhou, Development of a $\mathrm{pH}$-activatable fluorescent probe and its application for visualizing cellular $\mathrm{pH}$ change. Analyst. 137 (2012) 4418-4420.

[29]K.K Yu, K. Li, J.T. Hou, J. Yang, Y.M. Xie, X.Q. Yu, Rhodamine based pH-sensitive "intelligent" polymers as lysosome targeting probes and their imaging applications in vivo. Polym. Chem. 5 (2014) 5804-5812.

[30] S.L. Shen, X.F. Zhang, S.Y. Bai, J.Y. Miao, B.X. Zhao, A novel ratiometric pH probe for extreme acidity based on FRET and PET, RSC Adv. 5 (2015) 13341-13346.

[31] X.F. Zhang, T. Zhang, S.L. Shen, J.Y. Miao, B.X. Zhao, A ratiometric lysosomal pH probe based on the naphthalimide-rhodamine system, J. Mater. Chem. B 3 (2015) 3260-3266.

[32] X.D. Liu, Y. Xu, R. Sun, Y.J. Xu, J.M. Lu, J.F. Ge, A coumarin-indole-based near-infrared ratiometric $\mathrm{pH}$ probe for intracellular fluorescence imaging. Analyst. 138 (2013) 6542-6550.

[33] Z. Yang, L.K. Hao, B. Yin, M.Y. She, M. Obst, Six-Membered Spirocycle Triggered Probe for Visualizing $\mathrm{Hg}^{2+}$ in Living Cells and Bacteria-EPS-Mineral Aggregates. Org. Lett. 15 (2013) 4334-4337.

[34] C.A. Parker, W.T. Rees, Correction of fluorescence spectra and measurement of fluorescence quantum efficiency. Analyst. 85 (1960) 587-600.

[35] J.H. Huang, Y.F. Xu, X.H. Qian, Rhodamine-based fluorescent off-on sensor for $\mathrm{Fe}^{3+}$ in aqueous solution and in living cells: 8-aminoquinoline receptor and 2:1 binding. Dalton Trans. 43 (2014) 59835989.

[36] M.Y.She, Z. Yang, B. Yin, J. Zhang, J. Gu, W.T. Yin, J.L. Li, G.F. Zhao, Z. Shi, A novel rhodamine-based fluorescent and colorimetric "off-on" chemosensor and investigation of the recognizing behavior towards $\mathrm{Fe}^{3+}$. Dyes pigm. 92 (2012) 1337-1343. 
[37] K. Li, Y. Xiang, X.Y. Wang, J. Li, R.R. Hu, A.J. Tang, B.Z. Tang, Reversible Photochromic System Based on Rhodamine B Salicylaldehyde Hydrazone Metal Complex. J. Am. Chem. Soc. 136 (2014) 1643-1649.

[38] Y. Ding, H. Zhu, X. Zhang, J.J. Zhu, B. Clemens, Rhodamine B derivative-functionalized upconversion nanoparticles for FRET-based $\mathrm{Fe}^{3+}$-sensing. Chem. Commun. 49 (2013) 7797-7799.

[39] J.F. Zhang, Y. Zhou, J. Yoon, Y. Kim, S.J. Kim, J.S. Kim, Naphthalimide Modified Rhodamine Derivative: Ratiometric and Selective Fluorescent Sensor for $\mathrm{Cu}^{2+}$ Based on Two Different Approaches. Org. Lett. 12 (2010) 3852-3855.

[40] B.M. Shubhra, K.B. Parimal, A Chemosensor Built with Rhodamine Derivatives Appended to an Aromatic Platform via 1,2,3-Triazoles: Dual Detection of Aluminum (III) and Fluoride/Acetate Ions. Inorg. Chem. 52 (2013) 1161-1163.

[41] S. Ohkuma, Y. Moriyama, T. Takano, Identification and characterization of a proton pump on lysosomes by fluorescein isothiocyanate-dextran fluorescence. Proc. Natl Acad. Sci. 79 (1982) 27582762.

[42] J.A. Mindell, Lysosomal acidification mechanisms. Annu. Rev. Physiol. 74 (2012) 69-86.

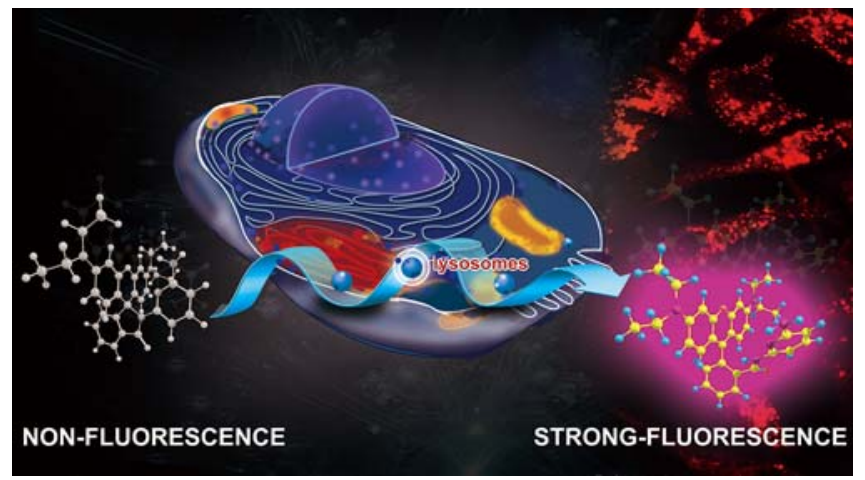

Graphical Abstracts 


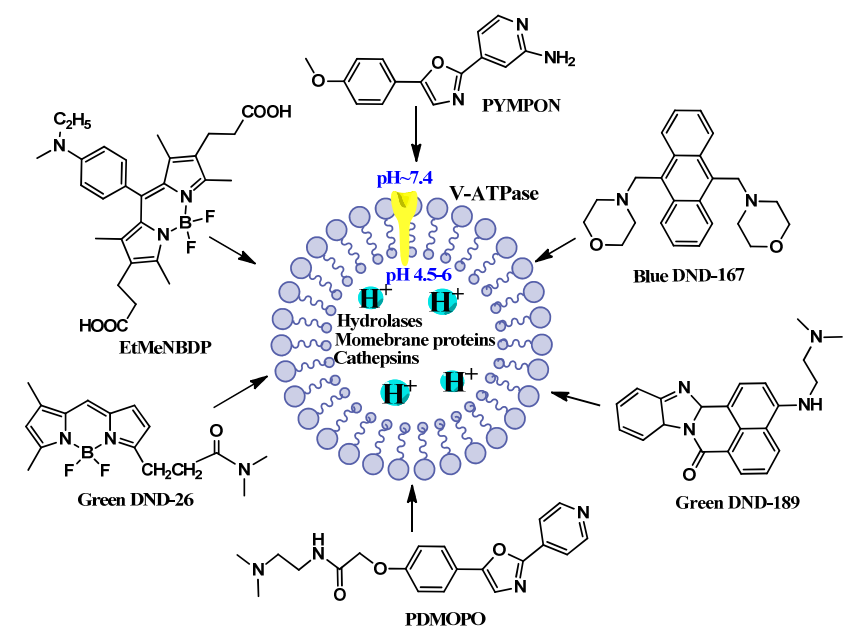

Figure 1. Some traditional lysosomal targeted probes base on PET (photo-induced electron transfer) process.

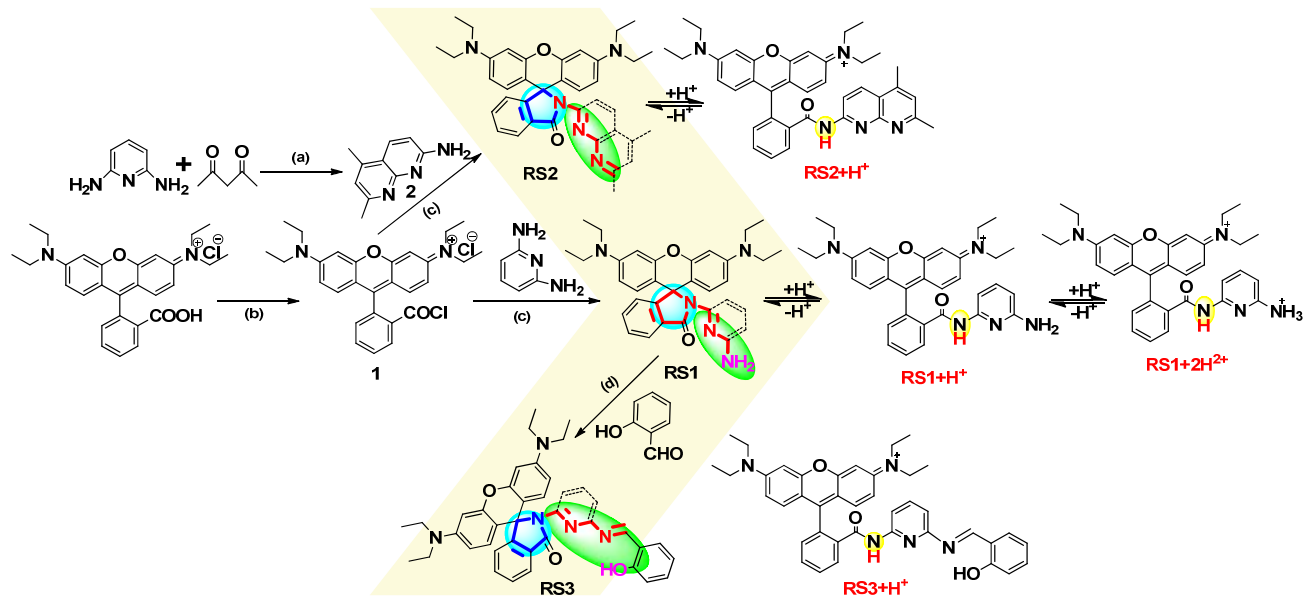

Scheme 1. The synthetic route of compound 2 and probe RS1, RS2 and RS3 and the recognition process. Reagents and conditions: (a) $\mathrm{H}_{3} \mathrm{PO}_{4}, 4$ h, $\mathrm{NaOH}$; (b) $\mathrm{POCl}_{3}$, 1,2-dichloroethane, reflux, 8 h; (c) $\mathrm{Et}_{3} \mathrm{~N}, \mathrm{CH}_{3} \mathrm{CN}$, reflux, 6 h; (d) $\mathrm{CH}_{3} \mathrm{CH}_{2} \mathrm{OH}$, reflux, 8 h. 


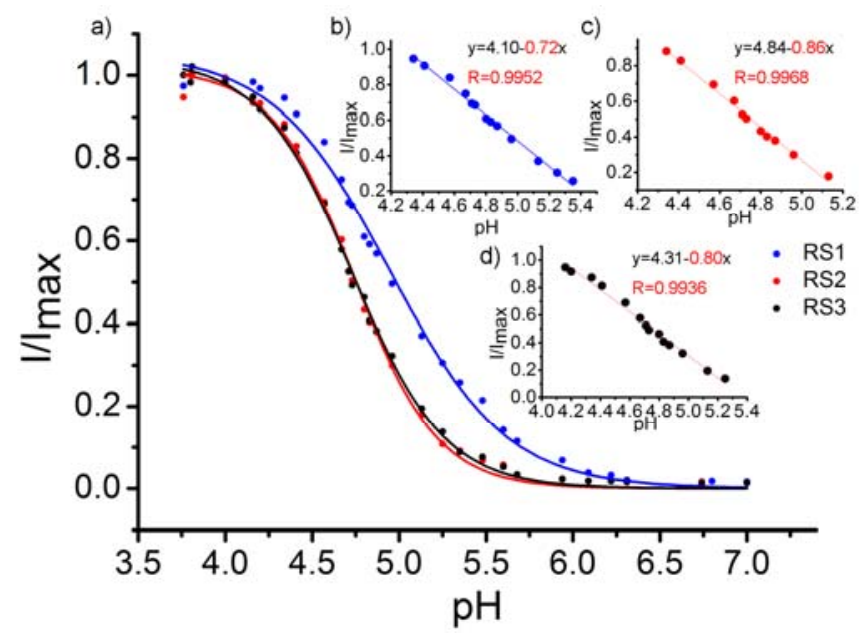

Figure 2. (a) Sigmoidal fitting of the maximum emission fluorescence intensity at $590 \mathrm{~nm}$ for RS1, RS2 and RS3. Inset: (b) the linearity of RS1 in the pH range of 4.34-5.35. (c) The linearity of RS2 in the $\mathrm{pH}$ range of 4.62-5.13. (d) The linearity of RS3 in the $\mathrm{pH}$ range of 4.16-5.25.

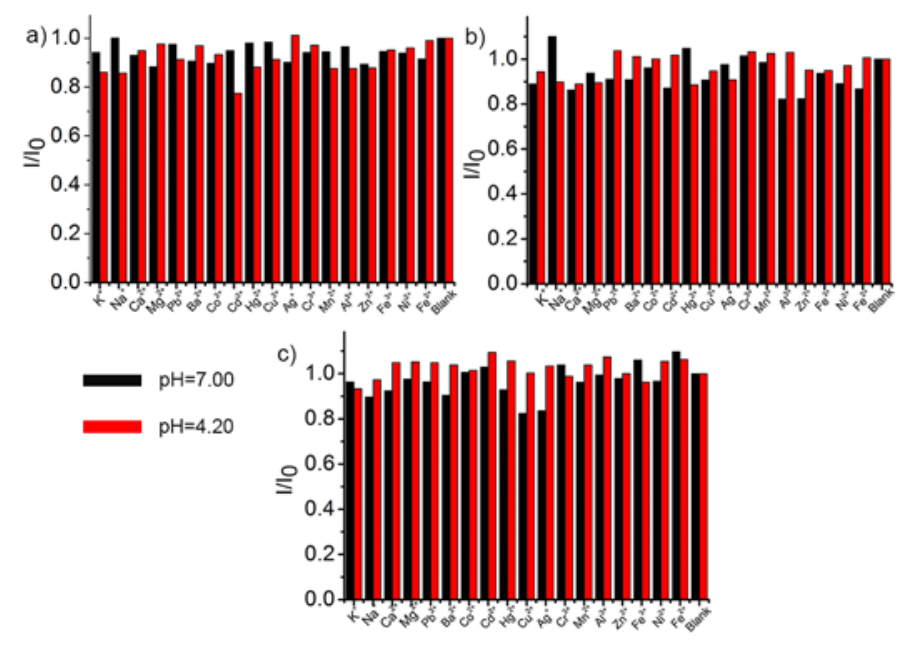

Figure 3. Relative fluorescence intensity in the presence of diverse metal ions at pH 4.20 and 7.00. a) $10 \mu \mathrm{M} \mathrm{RS} 1$ in B-R buffer-EtOH aqueous solvents, (v/v = 8:2) at $590 \mathrm{~nm}$. b) $10 \mu \mathrm{M} \mathrm{RS} 2$ in BR buffer-EtOH aqueous solvents, $(\mathrm{v} / \mathrm{v}=8: 2)$ at $590 \mathrm{~nm}$. c) $10 \mu \mathrm{M}$ RS3 in B-R buffer-EtOH aqueous solvents, $(\mathrm{v} / \mathrm{v}=8: 2)$ at $590 \mathrm{~nm} \cdot \mathrm{Na}^{+}(150 \mathrm{mM}) ; \mathrm{K}^{+}(10 \mathrm{mM}) ; \mathrm{Ca}^{2+}, \mathrm{Mg}^{2+}, \mathrm{Zn}^{2+}, \mathrm{Fe}^{2+}, \mathrm{Co}^{2+}(5$ $\mathrm{mM}$ ); other ions with $0.1 \mathrm{mM}$. 


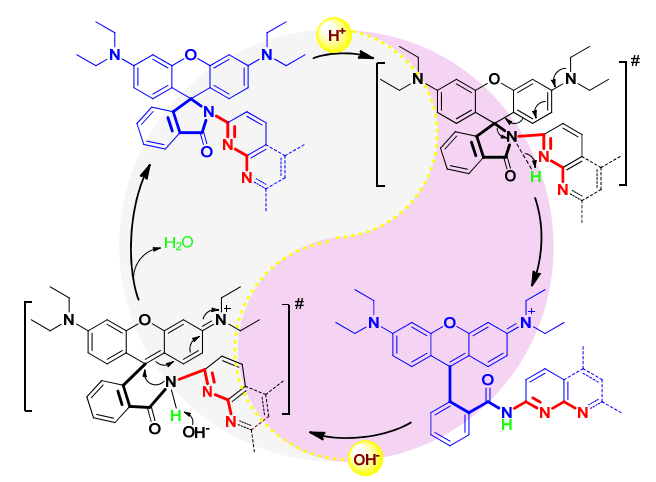

Figure 4. The reversibility mechanism of probe $\mathbf{R S 2}$.
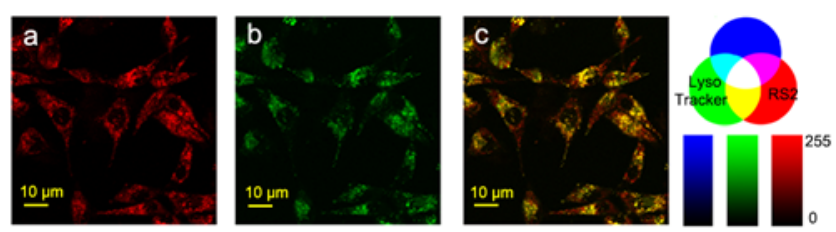

Figure 5. (a) Fluorescence microscope images of living A549 cells co-stained with $4 \mu \mathrm{M}$ RS2 (red channel); (b) Green emission from LysoTracker Green $0.5 \mu \mathrm{M}$ DND-26 (red channel); (c) Merging of (a) and (b), areas of co-localization are shown in yellow. 


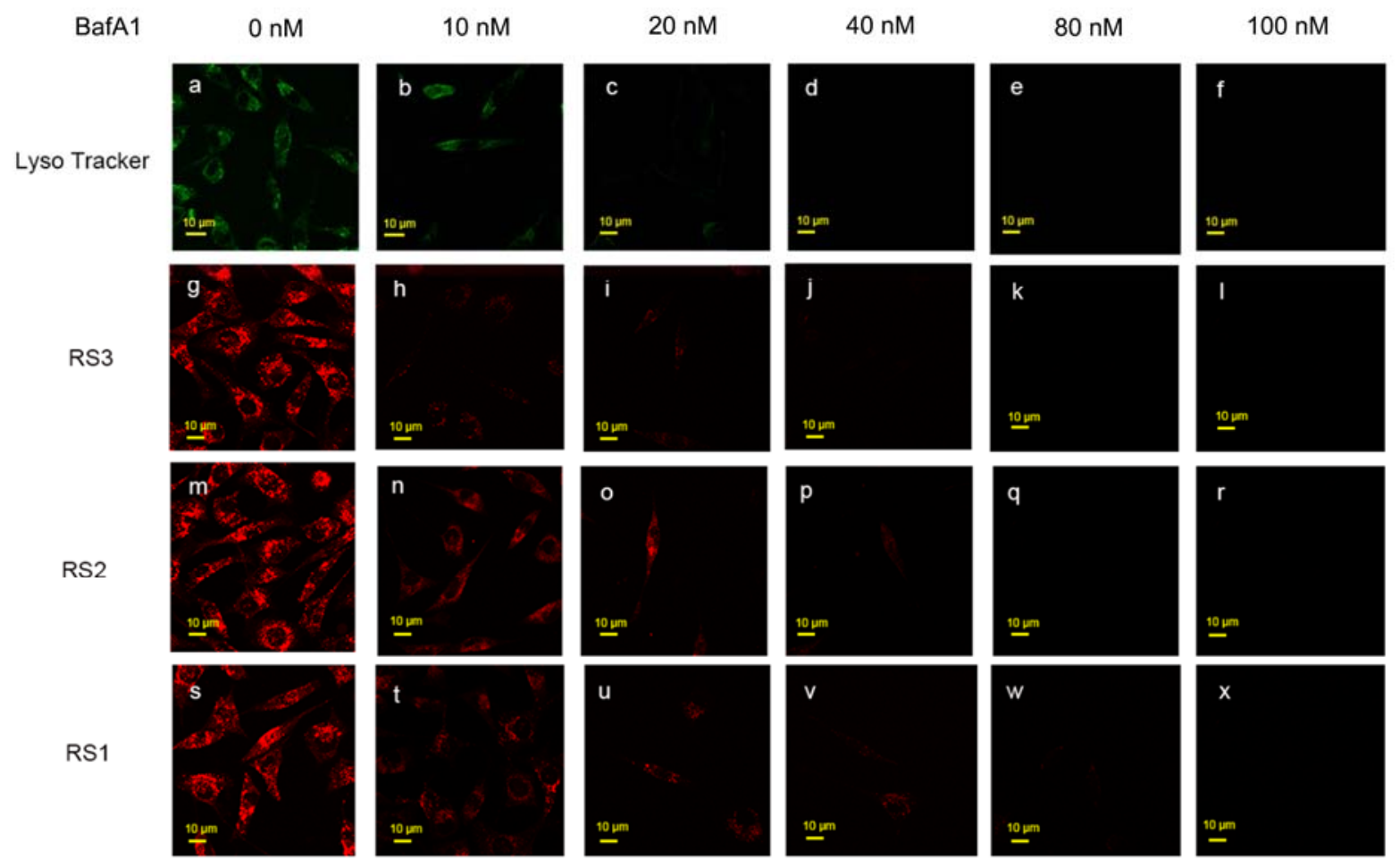

Figure 6. Lysosomal pH dependent staining of A549 cells with RS1 (10 $\mu \mathrm{M})$, RS2 (10 $\mu \mathrm{M})$, RS3 $(10 \mu \mathrm{M})$ and LysoTracker green DND-26(0.5 $\mu \mathrm{M})$. A549 cells pretreated with or without Bafilomycin A1 about $5 \mathrm{~h}$ and then analyzed by confocal maaaaicroscopy. The intracellular of RS2, RS3 fluorescence is shown in red and that of LysoTracker green DND-26 is shown in green. 


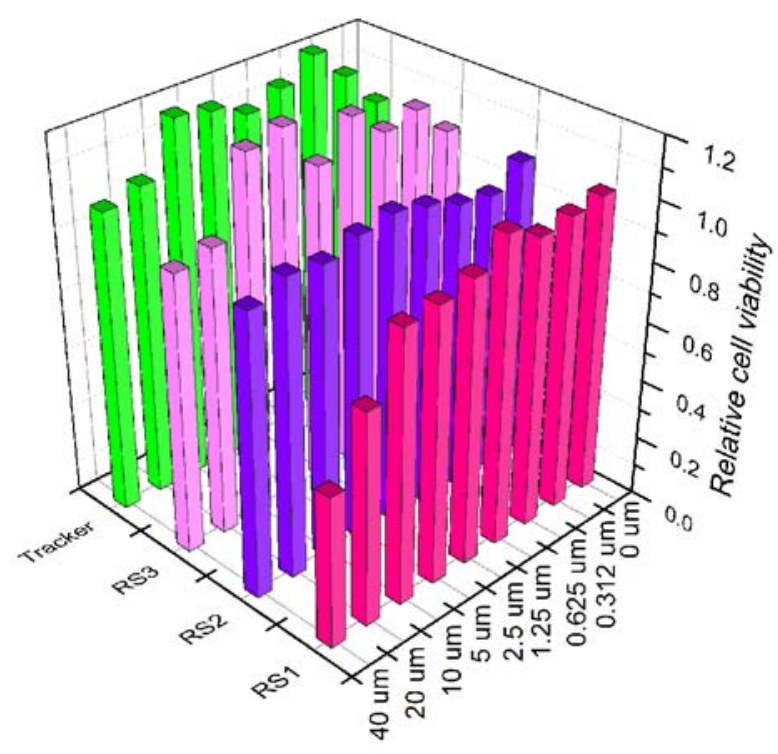

Figure 7. Cell toxicity of LysoTracker, RS1, RS2, and RS3 on A549 cells. 